\title{
Large-Time Behavior of Magnetohydrodynamics with Temperature-Dependent Coefficients
}

\author{
Song Dandan ${ }^{1}$ \\ ${ }^{1}$ Guizhou University
}

September 24, 2021

\begin{abstract}
In this paper, we investigate the initial and boundary value problem of a planar magnetohydrodynamic system with temperaturedependent coefficients of transport, heat conductivity, and magnetic diffusivity coefficients. When all of the relative coefficients are exponentially related to the temperature, the existence and uniqueness of the global-in-time non-vacuum strong solutions are proven under some special assumptions. At the same time, the nonlinearly exponential stability of the solutions is obtained. In fact, the initial data could be large if the positive growth exponent of viscosity is small enough.
\end{abstract}

\section{Hosted file}

Large-Time Behavior of Magnetohydrodynamics with Temperature Dependent Coefficients.pdf available at https://authorea.com/users/435005/articles/538322-large-time-behavior-ofmagnetohydrodynamics-with-temperature-dependent-coefficients 